\title{
CARIS Life Sciences
}

National Cancer Institute

\section{Source}

National Cancer Institute. CARIS Life Sciences. NCI Thesaurus. Code C133728.

A biotechnology company that specializes in molecular profiling of cancers and identification of targets for treatment. 